\title{
Erratum
}

\section{Erratum to: A Finite Dimensional Integrable System Arising in the Study of Shock Clustering}

\section{Luen-Chau Li (i)}

Department of Mathematics, Pennsylvania State University, University Park, PA 16802, USA.

E-mail: luenli@math.psu.edu

Received: 18 December 2016 / Accepted: 9 February 2017

Published online: 10 March 2017 - @ Springer-Verlag Berlin Heidelberg 2017

Commun. Math. Phys. 340, 1109-1142 (2015)

It has come to the author's attention recently that the variables $\phi_{r 0}(L), r=1, \ldots, N-1$, as defined on the second line of (4.35) in [L] simply do not work as claimed in Theorem 4.10 (b) and Proposition 4.13 (b) of that work. Regrettably, this is due to overlooking a term in the proof of Theorem 4.10 (b). The purpose of this erratum is to provide the correct definition of the $\phi_{r 0}$ 's to make things work and what is required is a divisor $D_{\tau}(L)$ different from the one in [L]. Indeed, this new divisor can also be used in constructing the variables that move linearly on the Jacobi variety of the curve. Therefore, while the variables $\phi_{r k}(L), 1 \leq k \leq r-1, r=2, \ldots, N$ in [L] are sound, we will replace them by corresponding quantities constructed by using $D_{\tau}(L)$ here because it gives us a uniform construction of all the angle variables.

Recall that $L \in \mathfrak{g}=\operatorname{gl}(N, \mathbb{R})$ and we have the Lax operator $L_{u}(h)=h u-L$. Let

$$
I(L ; h, z)=\operatorname{det}\left(L_{u}(h)-z I\right)=\sum_{r=0}^{N} \sum_{k=0}^{r} I_{r k}(L) h^{k} z^{N-r}
$$

and denote its homogenization by $\widetilde{I}(L ; \zeta, h, z)$, i.e.,

$$
\widetilde{I}(L ; \zeta, h, z)=\operatorname{det}(h u-\zeta L-z I) .
$$

Then we have the projective curve

$$
C(L)=\left\{[\zeta: h: z] \in \mathbb{C P}^{2} \mid \widetilde{I}(L ; \zeta, h, u)=0\right\}
$$

with affine part $C_{a}(L): I(L ; h, z)=0$. In the notation of [L], we will work with $L \in \mathcal{G} \cap \mathcal{U}$ satisfying the additional genericity assumption (GA1). Also, recall that 
(h) $=P_{+}-P_{-}, P_{ \pm}=\sum_{i=1}^{N} P_{ \pm}^{i}$. In order to define the divisor alluded to above, we have to consider eigenvectors of the transpose $L_{u}(h)^{T}$ of the Lax operator. If for $P=[\zeta: h: z] \in C(L)$, we let $\mathcal{M}(L, P)=h u-\zeta L-z I$, then $f_{\tau}(P)=\operatorname{ker} \mathcal{M}(L, P)^{T}$ defines the holomorphic eigenvector map $f_{\tau}: C(L) \longrightarrow \mathbb{C P}^{N-1}$. Let

$$
H=\left\{\left[z_{1}: \cdots: z_{N}\right] \in \mathbb{C P}^{N-1} \mid z_{1}+\cdots+z_{N}=0\right\},
$$

then the divisor $D_{\tau}(L)=f_{\tau}^{*} H$ is well-defined and is of degree $\frac{N(N-1)}{2}=g+N-1$, where $g$ is the genus of $C(L)$. Recall that [L]

$$
\begin{aligned}
& \omega_{r+1,-1}=\frac{z^{N-r-1}}{h I_{z}(h, z)} d h, r=1, \ldots, N-1, \\
& \omega_{r+1, k-1}=\frac{h^{k-1} z^{N-r-1}}{I_{z}(L ; h, z)} d h, 1 \leq k \leq r-1, r=2, \ldots, N-1 .
\end{aligned}
$$

As replacement of the variables in [L], we define

$$
\begin{aligned}
& \phi_{r 0}(L)=\int_{D_{0}(L)}^{D_{\tau}(L)} \omega_{r+1,-1}, r=1, \ldots, N-1, \\
& \phi_{r k}(L)=\int_{D_{0}(L)}^{D_{\tau}(L)} \omega_{r+, k-1}, \quad 1 \leq k \leq r-1, r=2, \ldots, N-1,
\end{aligned}
$$

where $D_{0}(L)=(g+N-1) P_{-}^{1}$, and in the definition of $\phi_{r 0}(L)$ above, the path of integration going from the point $P_{-}^{1}$ to the points in $D_{\tau}(L)$ have to avoid the points in $P_{+}^{i}, i=1, \ldots, N$.

\section{Theorem 4.10'.}

(a) $\left\{\phi_{r k}, I_{r^{\prime} k^{\prime}}\right\}_{R}(L)=-\delta_{k k^{\prime}} \delta_{r^{\prime}, r+1}, 1 \leq k \leq r-1, r=2, \ldots, N-1,0 \leq k^{\prime} \leq r-2$, $r^{\prime}=2, \ldots, N$.

(b) $\left\{\phi_{r 0}, I_{r^{\prime} k^{\prime}}\right\}_{R}(L)=-\delta_{k^{\prime} 0} \delta_{r^{\prime}, r+1}, r=1, \ldots, N-1,0 \leq k^{\prime} \leq r-2, r^{\prime}=2, \ldots, N$.

Proof. We will give the proof of part (b). For this purpose, recall that [L] we pick $h_{0} \in(-1,1), z_{0} \in \mathbb{R}$ such that $\left[1: h_{0}: z_{0}\right] \notin C(L)$, and we set $H_{h_{0}, z_{0}}(L)=$ $\log \operatorname{det}\left(L_{u}\left(h_{0}\right)-z_{0} I\right)$. Let $P_{i}=\left[1: h_{0}: z_{i}\left(h_{0}\right)\right] \in C(L), i=1, \ldots, N$. To compute $\left\{\phi_{r 0}, H_{h_{0}, z_{0}}\right\}_{R}(L)$, it suffices to evaluate it on an open dense subset of $L$ satisfying the following conditions:

(i) the points of $D_{\tau}(L)$ are distinct, $\operatorname{supp} D_{\tau}(L) \subset C_{a}(L)$,

(ii) $\operatorname{supp} D_{\tau}(L) \cap\left\{I_{z}=0\right\}=\emptyset$,

(iii) $\operatorname{supp} D_{\tau}(L) \cap\left\{P_{i}\right\}_{i=1}^{N}=\emptyset$,

(iv) $\left\{P_{i}\right\}_{i=1}^{N} \cap\left\{I_{z}=0\right\}=\emptyset$.

For small values of $t$, let $L(t)$ be the solution of the Hamiltonian flow generated by $H_{h_{0}, z_{0}}$ with initial condition $L(t=0)=L$, and let $D_{\tau}(t)=\left(f_{\tau}^{t}\right)^{*} H=\sum_{j=1}^{g+N-1} Q_{j}(t)$, where $f_{\tau}^{t}: C(L) \longrightarrow \mathbb{C P}^{N-1}$ is the holomorphic eigenvector map defined by $f_{\tau}^{t}(P)=$ 
$\operatorname{Ker} \mathcal{M}(L(t), P)^{T}$. Let $Q_{j}(t)=\left[1: h_{j}(t): z_{j}(t)\right], Q_{j}=Q_{j}(0), j=1, \ldots, g+N-1$. Then we have

$$
\begin{aligned}
\left\{\phi_{r 0}, H_{h_{0}, z_{0}}\right\}_{R}(L) & =\left.\frac{d}{d t}\right|_{t=0} \phi_{r 0}(L(t)) \\
& =\left.\sum_{j=1}^{g+N-1} \frac{z_{j}^{N-r-1}}{h_{j} I_{z}\left(h_{j}, z_{j}\right)} \frac{d h_{j}(t)}{d t}\right|_{t=0},
\end{aligned}
$$

where $h_{j}=h_{j}(0), z_{j}=z_{j}(0), j=1, \ldots, g+N-1$. To compute $\left.\frac{d h_{j}(t)}{d t}\right|_{t=0}$, consider a representative $f_{\tau}(h, t)$ of the kernel map $f_{\tau}^{t}$ in a neighborhood of $h_{j}$ such that $\left(e, f_{\tau}\left(h_{j}(t), t\right)\right)=0$ for small values of $t$. (Here $e$ is the vector in $\mathbb{C}^{N}$ with all components equal to 1.) Differentiating this relation with respect to $t$ at $t=0$, we have

$$
0=\left(e,\left.\frac{\partial f_{\tau}}{\partial h}\left(h_{j}, 0\right) \frac{d h_{j}(t)}{d t}\right|_{t=0}+\frac{\partial f_{\tau}}{\partial t}\left(h_{j}, 0\right)\right) .
$$

On the other hand, by differentiating

$$
L_{u}(h, t)^{T} f_{\tau}(h, t)=z f_{\tau}(h, t), \quad L_{u}(h, t)=h u-L(t),
$$

we obtain

$$
\left(L_{u}(h, t)^{T}-z I\right)\left(\frac{\partial f_{\tau}}{\partial t}+B(h)^{T} f_{\tau}(h, t)\right)=0,
$$

where

$$
B(h)=\Pi_{\mathfrak{m}}\left(L_{u}\left(h_{0}\right)-z_{0} I\right)^{-1}-\frac{h}{h-h_{0}}\left(L_{u}\left(h_{0}\right)-z_{0} I\right)^{-1}
$$

from Lemma 4.9 in [L]. Consequently,

$$
\begin{aligned}
\left(e, \frac{\partial f_{\tau}}{\partial t}\left(h_{j}, 0\right)\right) & =-\left(e, B\left(h_{j}\right)^{T} f_{\tau}\left(h_{j}, 0\right)\right) \\
& =\frac{h_{j}}{h_{j}-h_{0}}\left(e,\left(L_{u}\left(h_{0}\right)-z_{0} I\right)^{-T} f_{\tau}\left(h_{j}, 0\right)\right)
\end{aligned}
$$

where we have used the property that $X e=0$ for $X \in \mathfrak{m}$. Hence it follows that

$$
\begin{aligned}
\left.\frac{d h_{j}(t)}{d t}\right|_{t=0} & =-\frac{\left(e, \frac{\partial f_{\tau}}{\partial t}\left(h_{j}, 0\right)\right)}{\left(e, \frac{\partial f}{\partial h}\left(h_{j}, 0\right)\right)} \\
& =-\frac{h_{j}}{h_{j}-h_{0}} \frac{\left(e,\left(L_{u}\left(h_{0}\right)-z_{0} I\right)^{-T} f_{\tau}\left(h_{j}, 0\right)\right)}{\left(e, \frac{\partial f_{\tau}}{\partial h}\left(h_{j}, 0\right)\right)} .
\end{aligned}
$$

Substitute this in (7) above, we find

$$
\begin{aligned}
& \left\{\phi_{r 0}, H_{h_{0}, z_{0}}\right\}_{R}(L) \\
& =-\sum_{j=1}^{g+N-1} \frac{z_{j}^{N-r-1}}{h_{j} I_{z}\left(h_{j}, z_{j}\right)} \frac{h_{j}\left(e,\left(L_{u}\left(h_{0}\right)-z_{0}\right)^{-T} f_{\tau}\left(h_{j}, 0\right)\right)}{\left(h_{j}-h_{0}\right)\left(e_{1}, \frac{\partial f_{\tau}}{\partial h}\left(h_{j}, 0\right)\right)} \\
& =-\sum_{j=1}^{g+N-1} \operatorname{Res}_{Q_{j}} \frac{h\left(e,\left(L_{u}\left(h_{0}\right)-z_{0} I\right)^{-T} f_{\tau}(h, 0)\right)}{\left(h-h_{0}\right)\left(e, f_{\tau}(h, 0)\right)} \omega_{r+1,-1} .
\end{aligned}
$$


Now the meromorphic 1-form

$$
\frac{h\left(e,\left(L_{u}\left(h_{0}\right)-z_{0} I\right)^{-T} f_{\tau}(h, 0)\right)}{\left(h-h_{0}\right)\left(e, f_{\tau}(h, 0)\right)} \omega_{r+1,-1}
$$

has poles at the points $P_{i}, i=1, \ldots, N$, supp $D_{\tau}(L)$ and possibly at $P_{-}^{i}, i=1, \ldots, N$. Thus

$$
\begin{aligned}
& \left\{\phi_{r 0}, H_{h_{0}, z_{0}}\right\}_{R}(L) \\
& =\sum_{i=1}^{N} \operatorname{Res}_{P_{i}} \frac{h\left(e,\left(L_{u}\left(h_{0}\right)-z_{0} I\right)^{-T} f_{\tau}(h, 0)\right)}{\left(h-h_{0}\right)\left(e, f_{\tau}(h, 0)\right)} \omega_{r+1,-1} \\
& \quad+\sum_{i=1}^{N} \operatorname{Res}_{P_{-}^{i}} \frac{h\left(e,\left(L_{u}\left(h_{0}\right)-z_{0} I\right)^{-T} f_{\tau}(h, 0)\right)}{\left(h-h_{0}\right)\left(e, f_{\tau}(h, 0)\right)} \omega_{r+1,-1} .
\end{aligned}
$$

To study the behaviour of the meromorphic 1 -form in a neighborhood of $P_{-}^{i}$, introduce local coordinate $\xi=h^{-1}$, then $z=\frac{u_{i}}{\xi}+O(1)$ and so

$$
\begin{aligned}
& \frac{h\left(e,\left(L_{u}\left(h_{0}\right)-z_{0} I\right)^{-T} f_{\tau}(h, 0)\right)}{\left(h-h_{0}\right)\left(e, f_{\tau}(h, 0)\right)} \omega_{r+1,-1} \\
& \quad \simeq\left(e,\left(L_{u}\left(h_{0}\right)-z_{0} I\right)^{-T} e_{i}\right) \frac{u_{i}^{N-r-1}}{\prod_{\mu \neq i}\left(u_{\mu}-u_{i}\right)} \xi^{r-1} d \xi .
\end{aligned}
$$

Since $r \geq 1$, we conclude that the meromorphic 1-form is holomorphic at $P_{-}^{i}, i=$ $1, \ldots, N$. On the other hand,

$$
\begin{aligned}
& \operatorname{Res}_{P_{i}} \frac{h\left(e,\left(L_{u}\left(h_{0}\right)-z_{0} I\right)^{-T} f_{\tau}(h, 0)\right)}{\left(h-h_{0}\right)\left(e, f_{\tau}(h, 0)\right)} \omega_{r+1,-1} \\
& =\frac{z_{i}\left(h_{0}\right)^{N-r-1}}{\left(z_{i}\left(h_{0}\right)-z_{0}\right) I_{z}\left(h_{0}, z_{i}\left(h_{0}\right)\right)} .
\end{aligned}
$$

Therefore, on assembling the calculations, we obtain

$$
\begin{aligned}
& \left\{\phi_{r 0}, H_{h_{0}, z_{0}}\right\}_{R}(L) \\
& \quad=\sum_{i=1}^{N} \frac{z_{i}\left(h_{0}\right)^{N-r-1}}{\left(z_{i}\left(h_{0}\right)-z_{0}\right) I_{z}\left(h_{0}, z_{i}\left(h_{0}\right)\right)} \\
& \quad=\lim _{R \rightarrow 0} \oint_{|z|=R} \frac{z^{N-r-1}}{\left(z-z_{0}\right) I\left(h_{0}, z\right)} \frac{d z}{2 \pi i}-\frac{z_{0}^{N-r-1}}{I\left(h_{0}, z_{0}\right)} . \\
& \quad=-\frac{z_{0}^{N-r-1}}{I\left(h_{0}, z_{0}\right)} .
\end{aligned}
$$

But

$$
\left\{\phi_{r 0}, H_{h_{0}, z_{0}}\right\}_{R}(L)=\frac{1}{I\left(h_{0}, z_{0}\right)} \sum_{r^{\prime}, k^{\prime}}\left\{\phi_{r 0}, I_{r^{\prime} k^{\prime}}\right\}_{R}(L) h_{0}^{k^{\prime}} z_{0}^{N-r^{\prime}},
$$

hence it follows from the above computation that $\left\{\phi_{r 0}, I_{r^{\prime} k^{\prime}}\right\}_{R}(L)=-\delta_{k^{\prime} 0} \delta_{r^{\prime}, r+1}$. 
From the above Poisson bracket relations and the expression of the Hamiltonian $H=$ $\frac{1}{2} \operatorname{tr}(L(F \circ L))$ in Proposition 4.6 of [L], the following proposition follows.

Proposition 4.13'. (a) $\left\{\phi_{r k}, H\right\}_{R}(L)=(-1)^{N+1}\left(\sum_{j=1}^{N} \frac{f\left(u_{j}\right)}{A_{j}\left(u_{j}\right)} u_{j}^{N-1-r}\right) \delta_{k, r-1}, 1 \leq$ $k \leq r-1, r=2, \ldots, N-1$.

(b) $\left\{\phi_{r 0}, H\right\}_{R}(L)=(-1)^{N+1}\left(\sum_{j=1}^{N} \frac{f\left(u_{j}\right)}{A_{j}\left(u_{j}\right)} u_{j}^{N-1-r}\right) \delta_{r-1,0}, r=1, \ldots, N-1$.

Note that the Poisson bracket relations in Theorem 4.10' and Proposition 4.13' above only differ from the ones in [L] by a sign. For this reason, the proof of functional independence of the $I_{r k}$ 's for $k=0, \ldots, r-1, r=1, \ldots, N$ on the open, dense subset of $\mathcal{G} \cap \mathcal{U}$ satisfying (GA1) in Corollary 4.11 of [L] remains the same.

Finally, we correct a typo in equation (4.30) of [L] as follows: the product $\prod_{j=1}^{N} \frac{x-\lambda_{j}}{\lambda_{i}-\lambda_{j}}$ on the left hand side should be replaced by

$$
\prod_{\substack{j=1 \\ j \neq i}}^{N} \frac{x-\lambda_{j}}{\lambda_{i}-\lambda_{j}} .
$$

\section{Reference}

[L] Li, L.-C.: A finite dimensional integrable system arising in the study of shock clustering. Commun. Math. Phys. 340, 1109-1142 (2015)

Communicated by P. Deift 